$\underline{\text { Review Article }}$

\title{
Moving from oral poliovirus vaccine to inactivated poliovirus vaccine in Sri Lanka: The rationale and challenges
}

\author{
*Priyantha Perera ${ }^{1}$ \\ Sri Lanka Journal of Child Health, 2017; 46: 70-74 \\ DOI: http://dx.doi.org/10.4038/sljch.v46i1.8227 \\ (Key words: Oral poliovirus vaccine, inactivated poliovirus vaccine, Sri Lanka)
}

\section{Background}

Sri Lanka is a low middle income country with a gross domestic product per capita of 3637.54 US dollars in $2016^{1}$. Geographically, it is situated in the South Asian region. Compared to other low middle income countries and countries in the region, health status indicators of Sri Lanka are comparable to developed countries. The neonatal mortality rate of 6.4 per thousand live births, infant mortality rate of 9.7 per thousand live births, maternal mortality rate of 22.3 per 100,000 live births and life expectancy of 69.9 years for males and 78.7 years for females are evidence for this ${ }^{2}$. Successes achieved in health indicators are directly related to free health facilities and free education resulting in high literacy rate which is $92.2 \%$ for males and $89.2 \%$ for females ${ }^{2}$. Immunization coverage in Sri Lanka for BCG is $97 \%$, for the third dose of pentavalent vaccine $99 \%$ and the third dose of poliovirus vaccine $99 \%{ }^{3}$. The highly efficient immunization programme of Sri Lanka, with an excellent coverage for all vaccines, has contributed immensely towards lowering mortality figures.

Poliomyelitis (polio) is a dreaded disease caused by a RNA virus belonging to Picornaviridae family. There are three serotypes of poliovirus, which differ antigenically and protection against one serotype does not provide protection against the others. Polioviruses with the potential of causing the disease are called wild polioviruses (WPV). Worldwide, WPV type-2 has not caused polio since $1999^{4}$. The last case due to WPV type-3 was reported in 2012 from Nigeria. In 2009, 23 countries reported one or more polio cases due to WPV1 or WPV3.

\section{${ }^{1}$ Senior Lecturer, Faculty of Medicine, University of Kelaniya and Honorary Consultant Paediatrician, Colombo North Teaching Hospital, Sri Lanka}

\section{*Correspondence: priyanthaprr@gmail.com}

The author declares that there are no conflicts of interest

Open Access Article published under the Creative

Commons Attribution CC-BY (CC) (i)

Globally, from an estimated 350,000 polio cases in more than 125 endemic countries in 1988, the number of cases has dropped to 74 in 2015. Failure to implement strategic policies has led to ongoing transmission of poliovirus in Afghanistan, Nigeria and Pakistan, which are still considered endemic. Failure to stop poliovirus transmission in these last remaining areas has the potential of causing as many as 200,000 new cases globally every year, within 10 years ${ }^{5}$. Out of the endemic countries, Afghanistan and Pakistan are in close proximity to Sri Lanka. From 2003 to 2009, the World Health Organisation (WHO) has recorded 133 events of polio due to imported WPV, in 29 previously polio-free countries. Risk of importation and subsequent spread was highest in the countries with low immunization coverage and in those bordering the endemic countries ${ }^{4}$. Sri Lanka was fortunate not to be in this list despite its close proximity to endemic countries, probably due to its high immunization coverage $^{3}$. The last case of polio detected in Sri Lanka was way back in 1993. However, till March 2014 it was not declared as a polio free country, mainly because of the risk of importing $\mathrm{WPV}^{6}$. First and the only reported case of vaccine associated paralytic polio in Sri Lanka was in $2013^{7}$.

Worldwide, the use of poliovirus vaccines have brought a drastic reduction in cases of polio. In 1955 Salk vaccine, the inactivated poliovirus vaccine (IPV), was licensed for use. In 1961 Sabin vaccine, the live attenuated oral poliovirus vaccine (OPV), was licensed as a monovalent vaccine. The trivalent OPV (tOPV) was licensed for use in 1963. Trivalent oral poliovirus (tOPV) vaccine was preferred by most of the countries and was the preferred poliovirus vaccine in the expanded programme on immunization as well as the polio eradication programme 4 . Bivalent OPV (bOPV) containing only type- 1 and type-3 was licensed in 2009. As type-2 vaccine reduces the efficacy of the other two vaccines, immunity against WPV1 and WPV3 is better with bOPV than OOPV $^{8}$. In addition, type- 2 vaccine accounts for about $40 \%$ of vaccineassociated paralytic poliomyelitis (VAPP) and $97 \%$ of the circulating vaccine derived polioviruses $(\mathrm{cVDPV})^{9}$. 
Moving from oral polio vaccine to inactivated polio.... Sri Lanka Journal of Child Health, 2017; 46: 70-74

In VAPP one of the attenuated viruses in OPV, spontaneous acquisition of virulence causing paralytic disease in recipients or in their close contacts. The estimated incidence of VAPP is 4 per $1,000,000$ birth cohort per year and is more likely after the first dose of OPV. In populations with low OPV coverage, vaccine strains can acquire the virulence and transmissibility of WPV. These can cause outbreaks of polio as $\mathrm{cVDPV}^{10}$.

\section{Current situation in Sri Lanka}

\section{Government sector}

Majority of Sri Lankan children are vaccinated at government clinics which are conducted free of charge. Up to 2015, at government clinics five tOPV doses were given at 2, 4, 6, 18 months and 5 years, along with other vaccine/vaccines due at that particular age ${ }^{11}$. As the number of paralytic polio cases caused by WPV declined drastically worldwide, previous eradication plans of WHO were changed to 'Polio Eradication and Endgame Strategic Plan', which addresses paralytic cases with WPV as well as cVDPV /VAPP ${ }^{12}$.

As type-2 vaccine is responsible for $97 \%$ of the cVDPV $+40 \%$ of VAPP, and no polio cases have occurred due to type-2 WPV since 1999, all countries using tOPV were to switch to bOPV in a synchronized manner as part of the polio endgame strategic $\operatorname{plan}^{13}$. On $30^{\text {th }}$ April 2016, Sri Lanka replaced tOPV with bOPV and the remaining tOPV stocks were removed from all clinics. According to the polio endgame strategic plan, countries should administer at least one dose of IPV to children at 14 weeks of age, before the tOPV- bOPV switch ${ }^{13}$. This was initiated in 2015, prior to the switch. As timing of this dose was made flexible according to the country's preference, Sri Lanka introduced the IPV dose at four months of age, along with other vaccines due at that age. The objective of the IPV dose is to boost the population immunity against type- 2 virus and thereby prevent a possible outbreak of polio due to WPV2, following removal of tOPV.

However, in Sri Lanka, due to a scarcity of supply of IPV, fractional doses of IPV (fIPV) are now given intradermally at 2 and 4 months instead of an intramuscular single $\operatorname{dose}^{14}$. Thus the current government polio vaccine schedule for Sri Lanka reads as: (OPV+IPV) - (OPV+ IPV) - OPV- OPVOPV. However studies have shown that fIPV is less effective compared to IPV ${ }^{15}$.

\section{Private sector}

Some children belonging to higher socio-economic strata in Sri Lanka receive vaccinations from private hospitals. As in the government sector, the vaccination process is closely monitored and the cold chain is specifically monitored by the drug company distributing the vaccines in Sri Lanka. In the private sector, children are usually given IPV, combined with five other vaccines as hexavalent vaccine. Hexavalent vaccine is given at 2, 4 and 6 months. Some clinicians administer another dose of hexavalent vaccine at 18 months, but others administer OPV along with diphtheria-pertussistetanus (DPT) vaccine. This is because DPT combined with IPV or IPV as a single vaccine (stand-alone IPV) is not available in the private sector of Sri Lanka. Similarly OPV is given at five years as DPT/DT used at 5 years is not combined with IPV. Hexavalent vaccine is not recommended at 5 years. Therefore the private sector polio vaccine schedule reads as (IPV-IPV-IPV-IPV-OPV) or (IPV- IPV-IPV-OPV-OPV). Stand-alone IPV is now available in both the government and private sectors, but only for the fourth month dose. Therefore, these diverse regimes are likely to continue until stand-alone IPV or combined vaccines with IPV for vaccination at 18 months and five years are made available in the private sector. Recently there was a shortage of hexavalent vaccine in the country and children who had one or two doses of IPV had to complete the course with OPV. Though private sector is also supposed to follow the national schedule of immunization, diverse schedules are been followed according to personal preferences and opinions at times.

\section{Analysis of the current scenario}

During the pre-eradication period and in countries with a higher risk of importing WPV, it is important to have OPV in their immunization programme. This is because OPV induces intestinal mucosal immunity, which interferes with replication and excretion of the WPV. Therefore OPV not only decreases the chances of infection but reduces the spread of WPV. As OPV mimics a natural infection, vaccine strains are excreted by the recipients boosting or inducing immunity among close contacts. This provides an important source of indirect immunization, especially in areas with low immunization coverage. Pharyngeal mucosal immunity is induced by IPV, but it has a much lower impact than OPV on replication and excretion of poliovirus in the lower intestinal tract.

When the risk of paralytic polio from WPV is lower than from paralysis caused by OPV, there is pressure on authorities to switch from OPV to IPV. However until transmission of WPV is interrupted globally there is a potential risk of importing WPV into polio free countries. For countries with a higher risk of importation and low coverage of immunization, the WHO recommends moving from tOPV to bOPV with a single dose of IPV at 14 weeks. Once there is no or minimal risk of WPV induced paralysis, OPV is totally removed from immunization schedules and replaced by IPV. The WHO aims to achieve this by the year 2020. Sri Lanka has adopted this strategy. 
Though the risk of importation of WPV is high, because of very good immunization coverage Sri Lanka could have adopted sequential schedules, which include both IPV and OPV. Moving from OPV to sequential schedules requires an immunization coverage of approximately $95 \%$. In sequential schedule one or two doses of IPV are followed by at least two doses of OPV. Thus a sequential schedule would read as: (IPV-OPVOPV) or (IPV-IPV-OPV-OPV). Main advantage of sequential schedules is the minimal risk of VAPP, while maintaining the high levels of intestinal mucosal immunity conferred by OPV. VAPP and cVDPV are mainly encountered following the first dose of OPV. When one or two IPV doses are given prior to OPV, the existing immunity will reduce the risk of cVDPV/VAPP. Effectiveness of sequential schedules has been clinically proven by many studies $^{16,17,18}$.

A number of countries in Central and Eastern Europe, Middle East, Far East, and South Africa have adopted sequential schedules ${ }^{4}$. It is not exactly clear why Sri Lanka did not adopt a sequential schedule. Although Sri Lanka presently enjoys very high immunization coverage, during the civil war that prevailed over three decades, there were serious disruptions in medical services in war affected areas. Both the rebels and government forces allowed poliovirus vaccination programmes to continue uninterrupted, but definite figures for immunization coverage in war affected areas was not known. Though the war is now over and civilian life is restored in these areas, among older generations there may be individuals without protection against polio. This and the cost of IPV would have prompted authorities to stick to the current schedule than adopting a sequential schedule.

At the initiation of the polio endgame strategic plan, Global Polio Eradication Initiative (GPEI) had ensured sufficient stand-alone IPV vaccines to all OPV using countries, to introduce a single dose to their immunization programmes. According to the initial global demand forecast, 580-624 million doses of IPV would be needed by 2018. Presently there are four manufacturers of stand-alone IPV: Sanofi-Pasteur, France, Serum Institute of India, GlaxoSmithKline, Belgium and Statens Serum Institute, Denmark. Sri Lanka is currently facing a shortage of IPV due to global scarcity and is not expecting new stocks till 2017. Therefore intradermal fractional dose (fIPV) has been introduced as a solution. At two and four months 0.1 $\mathrm{ml}$ of IPV will be administered intra-dermally to the left upper arm. Administering two doses of fIPV instead of one may counteract the findings of studies which show less effectiveness of fIPV over IPV ${ }^{14}$.
Issues for the future

There is a need to change the immunization schedule of a country with changing scientific knowledge, shifting epidemiology and with invention of new vaccines. However, there have been too many changes, too frequently, in the Sri Lankan immunization schedule recently. This is not healthy as it might cause confusion among health workers and loss of faith in parents. Current strength of the Sri Lankan immunization programme is its high coverage, which is mainly based on faith of parents and dedication of grass root healthcare workers. We should avoid any actions that will undermine these strengths of our immunization programme.

One should respect the right of parents who wish to get their child vaccinated from the private sector. However, ideally, the schedules and types of vaccines in the government sector and private sector should be the same or else there should be a universal immunization schedule for the private sector as in the government sector. Different schedules used in different institutions and different individuals is causing a certain degree of confusion at present.

Cost of IPV which is considerably higher than that of OPV is an important factor to be considered in a country like Sri Lanka with a limited health budget. In the US market one dose from a 10 dose vial of IPV will cost around 12 US\$. Hopefully organizations like UNICEF will provide IPV to countries like Sri Lanka at a considerably reduced price. With the aim of global eradication of polio by 2018, bOPV is expected to be replaced by IPV around 2020. Sri Lanka and other developing countries will need to establish enough funds to support an uninterrupted supply of IPV.

Cold chain maintenance and storage is the other issue as IPV will take up some additional space. However IPV is a more heat stable vaccine and should not be frozen. If combination vaccines containing IPV is introduced it will reduce the burden on storage. As DPT (whole cell pertussis) vaccine, currently used in Sri Lanka is not combined with IPV, it would mean moving to DaPT (acellular pertussis) with IPV. This will reduce the side effects encountered by the child because majority of side effects are due to cellular pertussis component and only one injection compared to two. However, such a move will increase the cost several fold.

Yet for all that, considering the successes of the Sri Lanka immunization programme that have been achieved so far, as long as an uninterrupted vaccine supply can be ensured, Sri Lanka can be expected to move smoothly from bOPV to IPV by the year 2020 . 


\section{References}

1. Sri Lanka GDP per capita, 1961-2016. Available from: www.tradingeconomics.com/srilanka/gdp-per-capita

2. Annual Health Statistics 2009/2010 Sri Lanka. Medical Statistics Unit, Ministry of Health, Nutrition and Indigenous Medicine, Sri Lanka. Available from: www.statistics.gov.lk/page.asp?page $=\mathrm{Hea}$ lth

3. Sri Lanka: WHO and UNICEF estimates of immunization coverage. Available from: www.who.int/immunization/monitering_s urveillance/data/lka.pdf

4. Weekly Epidemiological Record. 4 June 2010, 85th year No. 23, 2010, 85, 213-228. Available from: http://www.who.int/we

5. World Health Organization. Poliomyelitis Fact Sheet, Updated April 2016. Available from:

www.who.int/mediacentre/factsheets/fs 11 4/en

6. WHO. South-East Asia Region certified polio-free. Available from: www.searo.who.int/mediacentre/releases/ 2014/pr1569/en

7. Perera P, Meththananda S. A Sri Lankan infant with vaccine associated acute flaccid paralysis. Ceylon Medical Journal 2012; 57(1):42-3. https://doi.org/10.4038/cmj.v57i1.4201 PMid: 22453711

8. Sutter RW, John TJ, Jain $H$, et al. Immunogenicity of bivalent types 1 and 3 oral poliovirus vaccine: a randomised, double-blind, controlled trial. Lancet 2010; 376(9753): 1682-8. https://doi.org/10.1016/S01406736(10)61 $230-5$

9. Kew $\mathrm{OM}$ et al. Vaccine-derived polioviruses and the endgame strategy for global polio eradication. Annual Review of Microbiology 2005; 59:587-635. https://doi.org/10.1146/annurev.micro.58. 030603.123625

PMid: 16153180
10. Risk assessment: frequency and burden of VAPP, cVDPV and iVDPV. In: Report of the interim meeting of the Technical Consultative Group (TCG) on the Global Eradication of Poliomyelitis: Geneva, 1314 November 2002.

11. Child Health Developmental Record. 2016. Ministry of health, Sri Lanka. Change of the polio vaccination schedule: Injectable inactivated polio vaccine (IPV): introduction of fractional dose IPV (fIPV). Ministry of Health, Nutrition \& Indigenous Medicine. Available from: http://www.epid.gov.lk/.../IPV/change_of_ ipv_vaccination.pdf

12. The Polio Eradication \& Endgame Strategic Plan 2013-2018. Available from: http://polioeradication.org/wpcontent/uplo ads/2016/07/2.8_8IMB.pdf

13. Global Polio Eradication Initiative. Fact Sheet - February 2015. Vaccine-associated paralytic polio (VAPP) and vaccinederived poliovirus (VDPV). Available from:

http://www.who.int/immunization/disease s/poliomyelitis/endgame_objective2/oral_ polio_vaccine/VAPPande-VDPVFactSheet -Feb2015.pdf

14. Change of the polio vaccination schedule: Injectable inactivated polio vaccine (IPV): introduction of fractional dose IPV (fIPV). Ministry of health, nutrition \& indigenous medicine.

http://www.epid.gov.lk/.../IPV/change_of ipv_vaccination.pdf Ministry of Health, Sri Lanka. Circular EPID/37/2016 dated $14 / 3 / 2016$

15. Anand A, Zaman K, Estívariz CF, Yunus M, Gary HE, Weldon WC. Early priming with inactivated poliovirus vaccine (IPV) and intradermal fractional dose IPV administered by a microneedle device: A randomized controlled trial. Vaccine 2015; 33(48): 6816-22.

https://doi.org/10.1016/j.vaccine.2015.09. 039

PMid: 26476367

16. Dömök I. Experiences associated with the use of live poliovirus vaccine in Hungary, 1959-1982. Review of Infectious Diseases 1984; 6(Suppl. 2):S413-S418. https://doi.org/10.1093/clinids/6.Suppleme nt $2 . \mathrm{S} 413$

PMid: 6740083 
17. Modlin $\mathrm{J}$ et al. Humoral and Mucosal Immunity in Infants Induced by Three Sequential Inactivated Poliovirus VaccineLive Attenuated Oral Poliovirus Vaccine Immunization Schedules. Journal of Infectious Diseases 1997; 175 (S1): S228 S234.

https://doi.org/10.1093/clinids/6.Suppleme nt 2. S413

PMid: 6740083

18. Von Magnus $\mathrm{H}$ et al. Vaccination with inactivated poliovirus vaccine and oral poliovirus vaccine in Denmark. Review of Infectious Diseases 1984; 6(Suppl. 2):S471-S474.

https://doi.org/10.1093/clinids/6.Suppleme nt 2.S471

PMid: 6740095 